\title{
Ultrafast Optical and Magneto-Optical Dynamics in Colossal-Magnetoresistance Manganites
}

\author{
V. Dorosinets ${ }^{a, b, *}$, P. RichteR ${ }^{a}$, G. JAKoB ${ }^{c}$ \\ AND H.G. RoskOs ${ }^{a}$ \\ ${ }^{a}$ Physikalisches Institut, J.W. Goethe-Universität, Frankfurt/Main 60054, Germany \\ ${ }^{b}$ Department of Semiconductor Physics, Belorussian State University \\ Minsk 220050, Republic of Belarus \\ ${ }^{c}$ Institut für Physik, J. Gutenberg-Universität Mainz, Mainz 60054, Germany

\begin{abstract}
Transient pump-probe reflectance measurements on $\mathrm{La}_{0.67} \mathrm{Ca}_{0.33} \mathrm{MnO}_{3} / \mathrm{MgO}$ and $\mathrm{La}_{0.67} \mathrm{Ca}_{0.33} \mathrm{MnO}_{3} / \mathrm{SrTiO}_{3}$ thin films reveal a hitherto unknown magnetization-dependent energy gap that determines the relaxation process of the optically excited charge carriers on the picosecond time scale. In the case of $\mathrm{La}_{0.67} \mathrm{Ca}_{0 .}{ }_{33} \mathrm{MnO}_{3} / \mathrm{SrTiO}_{3}$, the subsequent reflectance dynamics at low temperatures is determined to be substrate-induced strain that drives the sample into a metastable state characterized by an increased number of paramagnetic domains.
\end{abstract}

PACS numbers: 78.47.+p

\section{Introduction}

The physics of the colossal-magnetoresistance (CMR) manganites is determined by a complex interplay between the spin, lattice, charge, and orbital degrees of freedom. A model compound of the CMR family, and the material studied in this paper, is $\mathrm{La}_{0.67} \mathrm{Ca}_{0.33} \mathrm{MnO}_{3}$, which undergoes both a metal-insulator transition and a magnetic phase transition from a paramagnetic to a ferromagnetically ordered state at the Curie temperature $T_{\mathrm{C}}$. At low temperatures, the spin polarization is close to unity making this material a representative for the class of half-metallic ferromagnets.

We investigate the reflectance dynamics following femtosecond optical excitation of the material in order to identify manifestations of the coupling of the spin, lattice, charge, and orbital degrees of freedom in the relaxation processes revealing themselves in the reflectance data.

*corresponding author; e-mail: dorosinets@phys.bsu.by 


\section{Experimental results}

Our measurements are performed on $180 \mathrm{~nm}$ thick $c$-axis-oriented epitaxial films of $\mathrm{La}_{0.67} \mathrm{Ca}_{0.33} \mathrm{MnO}_{3}$ (LCMO) grown by pulsed $\mathrm{KrF}$ laser-sputtering deposition on $\mathrm{MgO}$ and $\mathrm{SrTiO}_{3}$ substrates (heater-block temperature: $950^{\circ} \mathrm{C}$ ). The temperature of the metal-insulator transition measured on a set of the samples is, on average, $240 \mathrm{~K}$ and does not reveal any noticeable dependence on the type of the substrate. The same holds for the temperature curves of the resistivity and magnetization. The peak value of the resistivity is $20 \mathrm{~m} \Omega \mathrm{cm}$ and the resistivity in the metallic state at low temperatures is in the range of $0.2-0.4 \mathrm{~m} \Omega \mathrm{cm}$. X-ray diffractometry yields only $(00 L)$ reflections (with $L$ integer) of the film indicating the excellent texture of the film. The $\operatorname{FWHM}(2 \Theta)$ value of the $(002)$ reflection amounts to 0.4 indicating the absence of large strain at room temperature.

A Ti:sapphire laser providing 60 fs laser pulses with a photon energy of $1.5 \mathrm{eV}$ at a repetition rate of $83 \mathrm{MHz}$ serves as light source for pump-probe measurements with a pump power, $P_{\mathrm{ex}}$, of $5 \mathrm{~mW}$ (fluence: $20 \mu \mathrm{J} / \mathrm{cm}^{2}$ ) and a probe power kept below $0.1 P_{\text {ex }}$.

We present transient reflectance change data taken on LCMO thin films focussing on the energy relaxation during the first few picoseconds after excitation. Figure 1 compares the reflectance-change transient $-\Delta R / R(t)$ of an $\mathrm{LCMO} / \mathrm{MgO}$
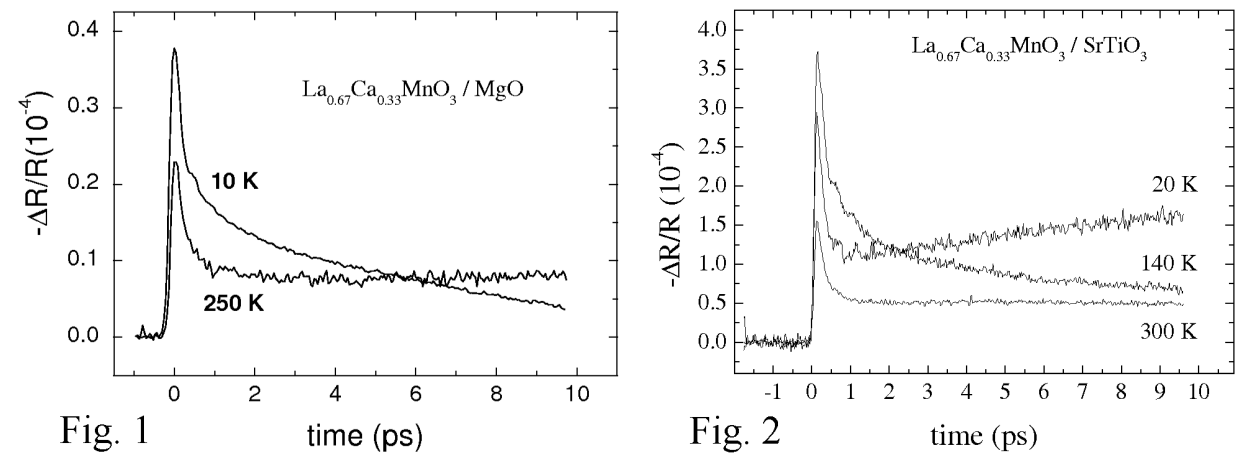

Fig. 1. Reflectance-change transients of $\mathrm{Laa}_{0.67} \mathrm{Ca}_{0.33} \mathrm{MnO}_{3} / \mathrm{MgO}$ film for two temperatures above, respectively below, $T_{\mathrm{C}}$. In the paramagnetic phase at $250 \mathrm{~K}$, the sample exhibits a single subpicosecond relaxation step. In the ferromagnetic state at $10 \mathrm{~K}$, one finds a subsequent relaxation step not present above $T_{\mathrm{C}}$.

Fig. 2. Reflectance-change transients of a $\mathrm{La}_{0.67} \mathrm{Ca}_{0.33} \mathrm{MnO}_{3} / \mathrm{SrTiO}_{3}$ film. Above the temperature of the structural phase transition of the $\mathrm{SrTiO}_{3}$ substrate $(105 \mathrm{~K})$, the relaxation is nearly identical to that of the $\mathrm{La}_{0.67} \mathrm{Ca}_{0.33} \mathrm{MnO}_{3} / \mathrm{MgO}$ film as shown in Fig. 1. At low temperatures, when the substrate has undergone the phase transition, the relaxation of the manganite film is drastically different. The slope of the second relaxation step has changed sign as seen in the curve measured at $20 \mathrm{~K}$. 
film $\left(T_{\mathrm{C}}=240 \mathrm{~K}\right)$ in the ferromagnetic phase at $10 \mathrm{~K}$ with that in the paramagnetic phase at $250 \mathrm{~K}$.

We analyze the decay of the $-\Delta R / R(t)$ curves by fitting the data with a sum of exponentials. The $250 \mathrm{~K}$ transient can be described by a single exponential with a decay time constant $\tau_{1}$ of $0.26 \mathrm{ps}$ followed by a plateau (or more correctly, a signal component with a nanosecond relaxation time constant). The corresponding curve at $10 \mathrm{~K}$, in contrast, requires two exponentials for a good fit, with time constants $\tau_{1}$ (of the same value as above $T_{\mathrm{C}}$ ) and $\tau_{2}=5.8 \mathrm{ps}$. The analysis of many more curves taken at various temperatures reveals that $\tau_{1}$ is temperature-independent, while the decay time constant $\tau_{2}$ rises (with falling temperature) from zero at $T_{\mathrm{C}}$ and above to values of $4-7 \mathrm{ps}$ at $T=10 \mathrm{~K}$. $\tau_{1}$, describing the relaxation right after the pump-induced reflectance drop, is attributed to thermalization in the charge degrees of freedom, i.e., to the transfer of the excess energy acquired by the optically excited charge carriers to the total carrier ensemble and to the lattice. The quasi-plateau, reached then for $T>T_{\mathrm{C}}$, indicates that the excited spot of the sample is at an elevated temperature which slowly approaches the ambient temperature on a nanosecond time scale much longer than the displayed time window of our measurements.

An interpretation of the additional relaxation step (represented by $\tau_{2}$ ) only present at $T<T_{\mathrm{C}}$ will be given below. An important aspect to be considered then is the observation that the $T$-dependencies of the relaxation time $\tau_{2}$ and of the magnetization of the LCMO film are very similar (data not shown). This finding leads us to suggest an effective magnetization-related energy gap to play an important role.

In the case of thin LCMO films, grown on $\mathrm{SrTiO}_{3}$ substrates, we find yet another behavior at reduced temperatures (see Fig. 2). While the transients above $T_{\mathrm{C}}$ and from there down to about $120 \mathrm{~K}$ show the same characteristics as observed in the case of $\mathrm{LCMO} / \mathrm{MgO}$ films, a remarkable change sets in when the temperature is reduced further. Below $90 \mathrm{~K}$, the slope of the $-\Delta R / R$ transients changes sign in a few picoseconds after arrival of the pump pulse. The $-\Delta R / R$ transients begin to rise again and do not continue to fall within the time window of the measurements.

Superimposed on all transient reflectance data of LCMO are strongly damped reflectance oscillations originating from coherent phonons. Fitting of the oscillation data yields a frequency $1 / \lambda=83 \mathrm{~cm}^{-1}(2.49 \mathrm{THz})$ which is likely to be the frequency of the rotational oscillation mode of the oxygen octahedra.

\section{Discussion}

The magnetic and transport properties of LCMO and other related manganites are determined by the $d$ electrons of $\mathrm{Mn}$. All electron spins on a given Mn ion are ferromagnetically aligned by a large Hund rule coupling. Mobile $e_{\mathrm{g}}$ electrons mediate the ferromagnetic interaction between $\mathrm{Mn}^{3+}$ and $\mathrm{Mn}^{4+}$ according 
to the double-exchange interaction (DE) model [1]. The strength of the ferromagnetic coupling between $\mathrm{Mn}^{3+}$ and $\mathrm{Mn}^{4+}$ ions depends strongly on the $\mathrm{Mn}-\mathrm{O}$ bond length and the Mn-O-Mn bond angle and any change in these parameters affects the electron hopping (scattering rate) significantly and hence the magnetic exchange interaction between $\mathrm{Mn}^{3+}$ and $\mathrm{Mn}^{4+}$. This ferromagnetic interaction competes with the coexisting antiferromagnetic superexchange interaction occurring via $t_{2 \mathrm{~g}}(\mathrm{Mn})-2 p_{\pi}(\mathrm{O})-t_{2 \mathrm{~g}}(\mathrm{Mn})$ as well as with the direct exchange. As a result of these competing interactions and because of the intrinsically random location of the alkaline-earth dopants, one expects the existence of nanometer-sized paramagnetic domains within the ferromagnetic matrix below $T_{\mathrm{C}}[2]$. A decrease in temperature diminishes the number of paramagnetic domains without ever having them disappear completely.

The decrease in the $-\Delta R / R(t)$ curve after the initial rise reflects a transformation of the preferentially metallic systems into a more insulating one as the sample temperature after laser excitation rises. This should go hand in hand with a loss of magnetization because of an increase in the total volume of the paramagnetic domains at the expense of the ferromagnetic domains. The buildup of paramagnetic domains may be described by an effective energy gap whose magnitude is determined by the degree of the ferromagnetic order. This gives rise to magnetization-related temperature dependence of the relaxation time for $\mathrm{LCMO} / \mathrm{MgO}$ films.

$\mathrm{SrTiO}_{3}$ exhibits a ferroelectric structural transformation at $105 \mathrm{~K}$ which induces strain within the LCMO film. The coincidence of this transition temperature with the temperature of the sign change in the reflectance transients points towards a high metastability of the phase-separated LCMO films. The metastability means that ferromagnetic and paramagnetic components are not in equilibrium and the state of the strained sample can be described by an effective temperature which exceeds the real temperature of the sample. Upon laser excitation, the effective temperature decreases, with the consequence that the signal rises representing a transition from a high-resistance state to a less resistive state. This should be accompanied by a transient increase in the magnetization which we at present have no means to measure directly. The pronounced coherent-phonon signature provides evidence that the metastable state is associated with a significant lattice distortion involving the oxygen octahedra (as proven by the observation of the rotational mode) and thus probably also involving the Jahn-Teller distortions.

\section{References}

[1] C. Zener, Phys. Rev. B 82, 403 (1951).

[2] E. Dagotto, T. Hotta, A. Moreo, Phys. Rep. 344, 1 (2001). 